\title{
Article
}

\section{General decay of the double dispersive wave equation with memory and source terms}

\author{
Mohamed Mellah \\ Faculty of Exact Sciences and Computer Science, Hassiba Benbouali University of Chlef, Chlef Algeria.; \\ m.mellah@univ-chlef.dz
}

Academic Editor: Qaisar Mehmood

Received: 11 November 2020; Accepted: 11 January 2021; Published: 20 January 2021.

Abstract: The double dispersive wave equation with memory and source terms $u_{t t}-\Delta u-\Delta u_{t t}+\Delta^{2} u-$ $\int_{0}^{t} g(t-\tau) \Delta^{2} u(\tau) d \tau-\Delta u_{t}=|u|^{p-2} u$ is considered in bounded domain. The existence of global solutions and decay rates of the energy are proved.

Keywords: Double dispersive wave equation, small data global Solution, general decay.

MSC: 35L35, 35L82, 35B40.

\section{Introduction}

$\mathbf{L}$

et $\Omega$ be a bounded domain in $\mathbb{R}^{N}(N \geq 1)$ with smooth boundary $\partial \Omega$. We consider the initial-boundary value problem:

$$
\begin{cases}u_{t t}-\Delta u-\Delta u_{t t}+\Delta^{2} u-g * \Delta^{2} u-\Delta u_{t}=|u|^{p-2} u, & x \in \Omega, t>0, \\ u=0, \quad \frac{\partial u}{\partial v}=0, & x \in \partial \Omega, t>0, \\ u(x, 0)=u_{0}(x), \quad u_{t}(x, 0)=u_{1}(x), & x \in \Omega,\end{cases}
$$

where $p>2$ and $v$ represents the unit outward normal to $\partial \Omega$. Here, $g(t)$ is a positive function that represents the kernel of the memory term, which will be specified in Section 2 and

$$
g * \Delta^{2} u(t)=\int_{0}^{t} g(t-\tau) \Delta^{2} u(\tau) d \tau
$$

The motivation of our work is due to the initial boundary problem of the double dispersive-dissipative wave equation with nonlinear damping and source terms

$$
\begin{cases}u_{t t}-\Delta u-\Delta u_{t t}+\Delta^{2} u-\Delta u_{t}+a\left|u_{t}\right|^{m-2} u_{t}=b|u|^{p-2} u, & x \in \Omega, t>0 \\ u=0, \quad \frac{\partial u}{\partial v}=0, & x \in \partial \Omega, t>0 \\ u(x, 0)=u_{0}(x), \quad u_{t}(x, 0)=u_{1}(x), & x \in \Omega, \\ a, b>0, & \end{cases}
$$

which has been discussed by Di and Shang [1] by considering the existence of global solutions and the asymptotic behavior of global solutions with $m \geq p$.

In the absence of the dispersive term and the nonlinear damping term, model (2) reduces to the following wave equation

$$
u_{t t}-\Delta u-\Delta u_{t t}-\Delta u_{t}=f(u) .
$$

Shang [2] studied the well-posedness, asymptotic behavior, and the finite time blow-up of the solutions under some suitable conditions on $f$ and for $N=1,2,3$. Zhang and $\mathrm{Hu}$ [3] showed the existence and the stability of global weak solutions. Xie and Zhong [4] obtained the existence of global attractors in $H_{0}^{1}(\Omega) \times$ $H_{0}^{1}(\Omega)$, where the nonlinear term $f$ satisfies a critical exponential growth assumption. Xu et al., [5] used the multiplier method to investigate the asymptotic behavior of solutions for (3). 
Mellah [6] considered the following initial-boundary value problem

$$
\begin{cases}u_{t t}-\Delta u+\Delta^{2} u-g * \Delta^{2} u+u_{t}=|u|^{p-1} u, & x \in \Omega, t>0 \\ u=0, \quad \frac{\partial u}{\partial v}=0, & x \in \partial \Omega, t>0, \\ u(x, 0)=u_{0}(x), \quad u_{t}(x, 0)=u_{1}(x), & x \in \Omega,\end{cases}
$$

in a bounded domain and $p>1$. He investigated the small data global weak solutions and general decay of solutions, respectively.

Motivated by previous works, it is interesting to prove that problem (1) has a global weak solution assuming small initial data. In addition, we show the general decay of solutions. The global solutions are constructed by means of the Galerkin approximations and the general decay is obtained by employing the technique used in [7].

\section{Preliminaries}

In this section, we present some materials needed in the proof of our main result. We use the following abbreviations; $\|\cdot\|_{p}=\|\cdot\|_{L^{p}(\Omega)}(1 \leq p \leq+\infty)$ denotes usual $L^{p}$ norm, $(\cdot, \cdot)$ denotes the $L^{2}$-inner product, and consider the Sobolev spaces $H_{0}^{1}(\Omega)$ and $H_{0}^{2}(\Omega)$ with their usual scalar products and norms. We also use the embedding $H_{0}^{1}(\Omega) \hookrightarrow L^{p}(\Omega)$ for $2<p \leq \frac{2 N}{N-2}$ if $N \geq 3$ or $2<p<\infty$ if $N=1,2$. In this case, the embedding constant is denoted by $C_{*}$, that is $\|u\|_{p} \leq C_{*}\|\nabla u\|_{2}$. We define

$$
Q(z)=\frac{1}{2} z^{2}-\frac{C_{*}^{p}}{p} z^{p}
$$

By the direct computation, we deduce that $Q$ is increasing in $\left[0, z_{0}\right]$, where $z_{0}=C_{*}^{\frac{p}{2-p}}$ is its unique local maximum.

Next, we give the assumptions for problem (1).

(G1) The relaxation function $g: \mathbb{R}_{+} \rightarrow \mathbb{R}_{+}$is a bounded $C^{1}$ function such that

$$
g(0)>0, \quad 0<\eta=1-\int_{0}^{\infty} g(\tau) d \tau \leq 1-\int_{0}^{t} g(\tau) d \tau=\eta(t)
$$

(G2) There exist positive constants $\xi_{1}$ and $\xi_{2}$ such that

$$
-\xi_{1} g(t) \leq g^{\prime}(t) \leq-\xi_{2} g(t) \quad \forall t \geq 0 .
$$

(G3) We also assume that

$$
2<p \leq \frac{2 N}{N-2} \text { if } N \geq 3 \text { and } p>2 \text { if } N=1,2,
$$

where $\lambda_{1}$ is the first eigenvalue of the following problem

$$
\Delta^{2} u=\lambda_{1} u \quad \text { in } \Omega, \quad u=\frac{\partial u}{\partial v}=0 \quad \text { in } \partial \Omega .
$$

Remark 1. [8] Assuming $\lambda_{1}$ is the first eigenvalue of the problem (4), we have

$$
\|\Delta u\|_{2}^{2} \geq \lambda_{1}\|\nabla u\|_{2}^{2}
$$

The energy associated with problem (1) is given by

$$
E(t)=\frac{1}{2}\left\|u_{t}\right\|_{2}^{2}+\frac{1}{2}\left\|\nabla u_{t}\right\|_{2}^{2}+\frac{1}{2}\left(1-\int_{0}^{t} g(\tau) d \tau\right)\|\Delta u\|_{2}^{2}+\frac{1}{2}\|\nabla u\|_{2}^{2}+\frac{1}{2}(g \circ \Delta u)(t)-\frac{1}{p}\|u\|_{p}^{p},
$$

for $u \in H_{0}^{2}(\Omega)$, where

$$
(g \circ \Delta u)(t)=\int_{0}^{t} g(t-\tau)\|\Delta u(\tau)-\Delta u(t)\|_{2}^{2} d \tau
$$


Now, we are in a position to state our main results.

\section{Main results}

In this section, we are going to obtain the existence of global weak solutions for problem (1) with the initial conditions $\left\|\nabla u_{0}\right\|_{2}<z_{0}$ and $E(0)<Q\left(z_{0}\right)$.

Theorem 1. Assume that (G1) - (G3) hold, and that $\left\{u_{0}, u_{1}\right\}$ belong to $H_{0}^{2}(\Omega) \times H_{0}^{1}(\Omega)$. Further assume that $\left\|\nabla u_{0}\right\|_{2}<z_{0}$ and $E(0)<Q\left(z_{0}\right)$. Then, problem (1) admits a global weak solution, which satisfies

$$
u \in L^{\infty}\left(0, \infty ; H_{0}^{2}(\Omega)\right), \quad u_{t} \in L^{\infty}\left(0, \infty ; H_{0}^{1}(\Omega)\right) .
$$

Moreover, the identity

$$
E(t)+\int_{0}^{t}\left\|\nabla u_{t}(\tau)\right\|_{2}^{2} d \tau-\frac{1}{2} \int_{0}^{t}\left(g^{\prime} \circ \Delta u\right)(\tau) d \tau+\frac{1}{2} \int_{0}^{t} g(\tau)\|\Delta u(\tau)\|_{2}^{2} d \tau=E(0),
$$

holds for $0 \leq t<\infty$. Also, for an increasing $C^{2}$ function $\zeta: \mathbb{R}^{+} \rightarrow \mathbb{R}^{+}$satisfying

$$
\zeta(0)=0, \quad \zeta_{t}(0)>0, \quad \lim _{t \rightarrow+\infty} \zeta(t)=+\infty, \quad \zeta_{t t}(t)<0 \quad \forall t \geq 0,
$$

and, if $\|g\|_{L^{1}(0, \infty)}$ is sufficiently small, we have for $\kappa>0$

$$
E(t) \leq E(0) e^{-\kappa \zeta(t)}, \quad \forall t \geq 0
$$

Remark 2. From (8) and (G2), we obtain

$$
\begin{aligned}
\frac{d}{d t} E(t) & =-\left\|\nabla u_{t}(t)\right\|_{2}^{2}+\frac{1}{2}\left(g^{\prime} \circ \Delta u\right)(t)-\frac{1}{2} g(t)\|\Delta u(t)\|_{2}^{2} \\
& \leq-\left\|\nabla u_{t}(t)\right\|_{2}^{2}-\frac{1}{2} \xi_{2}(g \circ \Delta u)(t)-\frac{1}{2} g(t)\|\Delta u(t)\|_{2}^{2} \leq 0 .
\end{aligned}
$$

\section{Proof of Theorem 1 (Main result)}

We divide the proof into two steps. In step 1, we prove the small data global existence of weak solutions by using the Faedo-Galerkin approximation and in step 2, we establish the general decay of energy employing the method used in [7].

\section{Step 1: Global existence of weak solutions}

Let $\left\{\omega_{j}\right\}_{j=1}^{\infty}$ be an orthogonal basis of $H_{0}^{2}(\Omega)$ with $\omega_{j}$ being the eigenfunction of the following problem:

$$
-\Delta \omega_{j}=\lambda_{j} \omega_{j}, \quad x \in \Omega, \quad \omega_{j}=0, \quad x \in \partial \Omega
$$

Let $V^{n}=$ Span $\left\{\omega_{1}, \omega_{2}, \cdots, \omega_{n}\right\}$. By the standard method of ODE, we know that there exists only one local solution

$$
u^{n}(t)=\sum_{j=1}^{n} b_{j}^{n}(t) \omega_{j}
$$

of the Cauchy problem as follows:

$$
\begin{gathered}
\int_{\Omega} u_{t t}^{n} \omega d x+\int_{\Omega} \nabla u^{n} \cdot \nabla \omega d x+\int_{\Omega} \nabla u_{t t}^{n} \cdot \nabla \omega d x+\int_{\Omega} \Delta u^{n} \cdot \Delta \omega d x \\
-\int_{0}^{t} g(t-\tau) \int_{\Omega} \Delta u^{n}(\tau) \cdot \Delta \omega d x d \tau+\int_{\Omega} \nabla u_{t}^{n} \cdot \nabla \omega d x-\int_{\Omega}\left|u^{n}\right|^{p-2} u^{n} \omega d x=0, \\
u^{n}(0)=u_{0}^{n} \rightarrow u_{0}, \quad \text { in } H_{0}^{2}(\Omega), \quad u_{t}^{n}(0)=u_{1}^{n} \rightarrow u_{1} \text { in } H_{0}^{1}(\Omega) .
\end{gathered}
$$


By the standard theory of ODE system, we prove the existence of solutions of problem (10)-(11) on some interval $\left[0, t_{n}\right), 0<t_{n}<T$ for arbitrary $T>0$, then, this solution can be extended to the whole interval $[0, T]$ using the first estimate given below.

\section{A Priori Estimates}

Setting $\omega=u_{t}^{n}(t)$ in (10), we have

$$
\begin{aligned}
& \frac{1}{2} \frac{d}{d t}\left\|u_{t}^{n}\right\|_{2}^{2}+\frac{1}{2} \frac{d}{d t}\left\|\nabla u_{t}^{n}\right\|_{2}^{2}+\frac{1}{2} \frac{d}{d t}\left\|\nabla u^{n}\right\|_{2}^{2}+\frac{1}{2} \frac{d}{d t}\left\|\Delta u^{n}\right\|_{2}^{2}-\frac{1}{p} \frac{d}{d t}\left\|u^{n}\right\|_{p}^{p}+\left\|\nabla u_{t}^{n}\right\|_{2}^{2} \\
& -\int_{0}^{t} g(t-\tau) \int_{\Omega} \Delta u^{n}(\tau) \cdot \Delta u_{t}^{n}(t) d x d \tau=0 .
\end{aligned}
$$

A direct computation shows that

$$
\begin{aligned}
& -\int_{0}^{t} g(t-\tau) \int_{\Omega} \Delta u^{n}(\tau) \cdot \Delta u_{t}^{n}(t) d x d \tau \\
& =\frac{1}{2} \frac{d}{d t}\left(g \circ \Delta u^{n}\right)(t)-\frac{1}{2} \frac{d}{d t}\left(\int_{0}^{t} g(\tau) d \tau\right)\left\|\Delta u^{n}(t)\right\|_{2}^{2}-\frac{1}{2}\left(g^{\prime} \circ \Delta u^{n}\right)(t)+\frac{1}{2} g(t)\left\|\Delta u^{n}(t)\right\|_{2}^{2} .
\end{aligned}
$$

Inserting (13) into (12) and integrating over $[0, t] \subset[0, T]$, we obtain

$$
\begin{aligned}
& \frac{1}{2}\left\|u_{t}^{n}\right\|_{2}^{2}+\frac{1}{2}\left\|\nabla u_{t}^{n}\right\|_{2}^{2}+\frac{\eta(t)}{2}\left\|\Delta u^{n}(t)\right\|_{2}^{2}+\frac{1}{2}\left\|\nabla u^{n}\right\|_{2}^{2}-\frac{1}{p}\left\|u^{n}\right\|_{p}^{p}+\int_{0}^{t}\left\|\nabla u_{t}^{n}(\tau)\right\|_{2}^{2} d \tau+\frac{1}{2}\left(g \circ \Delta u^{n}\right)(t) \\
& -\frac{1}{2} \int_{0}^{t}\left(g^{\prime} \circ \Delta u^{n}\right)(\tau) d \tau+\frac{1}{2} \int_{0}^{t} g(\tau)\left\|\Delta u^{n}(\tau)\right\|_{2}^{2} d \tau=E^{n}(0) .
\end{aligned}
$$

From assumption (G3) and the Sobolev embedding, we have

$$
\left\|u^{n}\right\|_{p}^{p} \leq C_{*}^{p}\left\|\nabla u^{n}\right\|_{2}^{p}
$$

and then we have

$$
\begin{aligned}
& \frac{1}{2}\left\|u_{t}^{n}\right\|_{2}^{2}+\frac{1}{2}\left\|\nabla u_{t}^{n}\right\|_{2}^{2}+\frac{\eta(t)}{2}\left\|\Delta u^{n}(t)\right\|_{2}^{2}+\mathcal{Q}\left(\left\|\nabla u^{n}\right\|_{2}^{2}\right)+\int_{0}^{t}\left\|\Delta u_{t}^{n}(\tau)\right\|_{2}^{2} d \tau+\frac{1}{2}\left(g \circ \Delta u^{n}\right)(t) \\
& -\frac{1}{2} \int_{0}^{t}\left(g^{\prime} \circ \Delta u^{n}\right)(\tau) d \tau+\frac{1}{2} \int_{0}^{t} g(\tau)\left\|\Delta u^{n}(\tau)\right\|_{2}^{2} d \tau \leq E^{n}(0) .
\end{aligned}
$$

By using the fact that

$$
-\int_{0}^{t}\left(g^{\prime} \circ \Delta u^{n}\right)(\tau) d \tau+\int_{0}^{t} g(\tau)\left\|\Delta u^{n}(\tau)\right\|_{2}^{2} d \tau \geq 0
$$

estimate (15) yields

$$
\frac{1}{2}\left\|u_{t}^{n}\right\|_{2}^{2}+\frac{1}{2}\left\|\nabla u_{t}^{n}\right\|_{2}^{2}+\frac{\eta(t)}{2}\left\|\Delta u^{n}(t)\right\|_{2}^{2}+\frac{1}{2}\left(g \circ \Delta u^{n}\right)(t)+\mathcal{Q}\left(\left\|\nabla u^{n}\right\|_{2}^{2}\right)+\int_{0}^{t}\left\|\nabla u_{t}^{n}(\tau)\right\|_{2}^{2} d \tau \leq E^{n}(0) .
$$

From $E(0)<\mathcal{Q}\left(z_{0}\right)$ and (11), it follows that

$$
E^{n}(0)<\mathcal{Q}\left(z_{0}\right)
$$

for sufficiently large $n$. We claim that there exists an integer $N$ such that

$$
\left\|\nabla u^{n}(t)\right\|_{2}^{2}<z_{0} \quad \forall t \in\left[0, t_{n}\right) \quad n>N .
$$

Suppose the claim is proved, then $\mathcal{Q}\left(\left\|\nabla u^{n}\right\|_{2}^{2}\right) \geq 0$ and from (16) and (17),

$$
\frac{1}{2}\left\|u_{t}^{n}\right\|_{2}^{2}+\frac{1}{2}\left\|\nabla u_{t}^{n}\right\|_{2}^{2}+\frac{\eta(t)}{2}\left\|\Delta u^{n}(t)\right\|_{2}^{2}+\frac{1}{2}\left(g \circ \Delta u^{n}\right)(t)+\int_{0}^{t}\left\|\nabla u_{t}^{n}(\tau)\right\|_{2}^{2} d \tau \leq E^{n}(0)<\mathcal{Q}\left(z_{0}\right),
$$


for sufficiently large $n$ and $0 \leq t<\infty$.

\section{Proof of the claim}

Suppose that (18) false, then for each $n>N$, there exists $t \in\left[0, t_{n}\right)$ such that $\left\|\nabla u^{n}(t)\right\|_{2} \geq z_{0}$. Note that from $\left\|\nabla u_{0}\right\|_{2}<z_{0}$ and (11) there exists $N_{0}$ such that

$$
\left\|\nabla u^{n}(0)\right\|_{2}<z_{0} \quad \forall n>N_{0} .
$$

Then by continuity there exits a first $\widetilde{t_{n}} \in\left[0, t_{n}\right)$ such that

$$
\left\|\nabla u^{n}\left(\widetilde{t_{n}}\right)\right\|_{2}=z_{0}
$$

from where

$$
\mathcal{Q}\left(\left\|\nabla u^{n}(t)\right\|_{2}\right) \geq 0 \quad \forall t \in\left[0, \widetilde{t_{n}}\right] .
$$

From $E(0)<\mathcal{Q}\left(z_{0}\right)$ and (19), there exists $N>N_{0}$ and $\gamma \in\left(0, z_{0}\right)$ such that

$$
\begin{aligned}
0 & \leq \frac{1}{2}\left\|u_{t}^{n}(t)\right\|_{2}^{2}+\frac{1}{2}\left\|\nabla u_{t}^{n}(t)\right\|_{2}^{2}+\frac{\eta(t)}{2}\left\|\Delta u^{n}(t)\right\|_{2}^{2}+\frac{1}{2}\left(g \circ \Delta u^{n}\right)(t)+\mathcal{Q}\left(\left\|\nabla u^{n}(t)\right\|_{2}^{2}\right) \\
& \leq \mathcal{Q}(\gamma) \quad \forall t \in\left[0, \widetilde{t_{n}}\right] \quad \forall n>N .
\end{aligned}
$$

The monotonicity of $\mathcal{Q}$ in $\left[0, z_{0}\right]$ implies that

$$
0 \leq\left\|\nabla u^{n}(t)\right\|_{2}^{2} \leq \gamma<z_{0} \quad \forall t \in\left[0, \widetilde{t_{n}}\right],
$$

in particular, $\left\|\nabla u^{n}(t)\right\|_{2}^{2}<z_{0}$, which is a contradiction to (20). From (19), we have

$$
\begin{array}{rlrl}
\left\|\Delta u^{n}\right\|_{2}^{2} & <\frac{2 \mathcal{Q}\left(z_{0}\right)}{\eta}, & & \leq t<\infty, \\
\left\|u_{t}^{n}\right\|_{2}^{2}<2 \mathcal{Q}\left(z_{0}\right), & & 0 \leq t<\infty, \\
\left\|\nabla u_{t}^{n}\right\|_{2}^{2} & <2 \mathcal{Q}\left(z_{0}\right), & 0 & \leq t<\infty, \\
\int_{0}^{t}\left\|\nabla u_{t}^{n}(\tau)\right\|_{2}^{2} d \tau & <\mathcal{Q}\left(z_{0}\right), & & 0 \leq t<\infty .
\end{array}
$$

Using Sobolev inequality, (5) and (21), it follows that

$$
\left\|u^{n}\right\|_{p}^{2} \leq C_{*}^{2}\left\|\nabla u^{n}\right\|_{2}^{2} \leq C_{*}^{2} \lambda_{1}^{-1}\left\|\Delta u^{n}\right\|_{2}^{2}<\frac{2 C_{*}^{2} \lambda_{1}^{-1} \mathcal{Q}\left(z_{0}\right)}{\eta}, \quad 0 \leq t<\infty .
$$

Moreover, by (25), we get

$$
\left|\left(\left|u^{n}\right|^{p-2} u^{n}, u^{n}\right)\right| \leq\left\|u^{n}\right\|_{p}^{p}<C_{*}^{p}\left(\frac{2 C_{*}^{2} \lambda_{1}^{-1} \mathcal{Q}\left(z_{0}\right)}{\eta}\right)^{\frac{p}{2}}, \quad 0 \leq t<\infty .
$$

Therefore, there exist $u, \chi$ and a subsequence still denotes $\left\{u_{n}\right\}$ such that

$$
\begin{gathered}
u_{n} \rightarrow u \text { weak star in } L^{\infty}\left(0, \infty ; H_{0}^{2}(\Omega)\right), \quad n \rightarrow+\infty, \\
u_{t}^{n} \rightarrow u_{t} \text { weak star in } L^{\infty}\left(0, \infty ; H_{0}^{1}(\Omega)\right), \quad n \rightarrow+\infty, \\
\left|u^{n}\right|^{p-2} u^{n} \rightarrow \chi \text { weak star in } L^{\infty}\left(0, \infty ; L^{\frac{p}{p-1}}(\Omega)\right), \quad n \rightarrow+\infty,
\end{gathered}
$$

Besides, from Lions-Aubin Lemma we also have

$$
u^{n} \rightarrow u \text { strongly in } L^{2}\left(0, \infty ; L^{2}(\Omega)\right), \quad n \rightarrow+\infty,
$$


and consequently, making use of the Lemma 1.3 in [9], we deduce

$$
\left|u^{n}\right|^{p-2} u^{n} \rightarrow \chi=|u|^{p-2} u \text { weak star in } L^{\infty}\left(0, \infty ; L^{\frac{p}{p-1}}(\Omega)\right), \quad n \rightarrow+\infty .
$$

Thus, we obtain that $u$ is a global weak of problem (1). In order to prove (7), we use the mean value theorem, we see that there exists $0<\theta_{n}<1$ such that

$$
\begin{aligned}
\left\|u^{n}\right\|_{p}^{p}-\|u\|_{p}^{p} & \leq p\left|\int_{\Omega}\right| u+\left.\theta_{n} u^{n}\right|^{p-2}\left(u+\theta_{n} u^{n}\right)\left(u^{n}-u\right) d x \mid \\
& \leq p\left\|u+\theta_{n} u^{n}\right\|_{p}^{p-1}\left\|u^{n}-u\right\|_{p} \\
& \leq c\left\|u^{n}-u\right\|_{p} \rightarrow 0 \text { as } n \rightarrow+\infty,
\end{aligned}
$$

and for each fixed $t>0$, we obtain

$$
\begin{aligned}
\left|(g \circ \Delta u)(t)-\left(g \circ \Delta u^{n}\right)(t)\right|= & \left|\int_{0}^{t} g(t-\tau)\|\Delta u(\tau)-\Delta u(t)\|_{2}^{2} d \tau-\int_{0}^{t} g(t-\tau)\left\|\Delta u^{n}(\tau)-\Delta u^{n}(t)\right\|_{2}^{2} d \tau\right| \\
\leq & \int_{0}^{t} g(t-\tau)\left\|\Delta u(\tau)-\Delta u^{n}(\tau)\right\|_{2}\left\|\Delta u(\tau)+\Delta u^{n}(\tau)\right\|_{2} d \tau \\
& +\int_{0}^{t} g(t-\tau)\left\|\Delta u(\tau)-\Delta u^{n}(\tau)\right\|_{2} d \tau\left\|\Delta u(t)+\Delta u^{n}(t)\right\|_{2} \\
& +\int_{0}^{t} g(t-\tau)\left\|\Delta u(\tau)+\Delta u^{n}(\tau)\right\|_{2} d \tau\left\|\Delta u(t)-\Delta u^{n}(t)\right\|_{2} \\
& +\int_{0}^{t} g(\tau) d \tau\left\|\Delta u(t)+\Delta u^{n}(t)\right\|_{2}\left\|\Delta u(t)-\Delta u^{n}(t)\right\|_{2} \\
\leq & c \int_{0}^{t} g(t-\tau)\left\|\Delta u(\tau)-\Delta u^{n}(\tau)\right\|_{2} d \tau \\
& +c \int_{0}^{t} g(\tau) d \tau\left\|\Delta u(t)-\Delta u^{n}(t)\right\|_{2} \rightarrow 0 \text { as } n \rightarrow+\infty .
\end{aligned}
$$

Thus, we have

$$
\lim _{n \rightarrow+\infty}\left\|u^{n}\right\|_{p}^{p}=\|u\|_{p}^{p}, \quad \lim _{n \rightarrow+\infty}\left(g \circ \Delta u^{n}\right)(t)=(g \circ \Delta u)(t) .
$$

From (11), it follows that $E^{n}(0) \rightarrow E(0)$ as $n \rightarrow+\infty$. Finally, taking $n \rightarrow+\infty$ in (14), we deduce that the energy identity (7) holds for $0 \leq t<\infty$.

\section{Step 2: General decay of the energy}

Here, we prove the energy decay estimate of the global solutions obtained in the previous section. To obtain the decay result, we use the following lemmas which are of crucial importance in the proof.

Lemma 1. Let $u \in L^{\infty}\left(0, \infty ; H_{0}^{2}(\Omega)\right)$ with $u_{t} \in L^{\infty}\left(0, \infty ; H_{0}^{1}(\Omega)\right)$ be the solution of $(1)$ and $E(0)<\mathcal{Q}\left(z_{0}\right)$, $\left\|\nabla u_{0}\right\|_{2}<z_{0}$, then we have

$$
0 \leq E(t) \leq C_{1}\left\|\nabla u_{t}\right\|_{2}^{2}+C_{2}\|\Delta u\|_{2}^{2}+\frac{1}{2}(g \circ \Delta u)(t),
$$

where $C_{1}=\frac{1}{2}\left(1+B^{2}\right), C_{2}=\frac{1}{2}\left(1+\lambda_{1}^{-1}\right)$ and $B$ is the optimal constant satisfying the Poincaré inequality $\left\|u_{t}\right\|_{2} \leq$ $B\left\|\nabla u_{t}\right\|_{2}$.

Proof. From $E(0)<\mathcal{Q}\left(z_{0}\right)$ and $\left\|\nabla u_{0}\right\|_{2}<z_{0}$, we can obtain $\mathcal{Q}\left(\|\nabla u(t)\|_{2}\right) \geq 0$ for $0 \leq t<\infty$. Thus we have

$$
\begin{aligned}
E(t) & =\frac{1}{2}\left\|u_{t}\right\|_{2}^{2}+\frac{1}{2}\left\|\nabla u_{t}\right\|_{2}^{2}+\frac{1}{2}\|\nabla u\|_{2}^{2}+\frac{1}{2}(g \circ \Delta u)(t)+\frac{1}{2}\left(1-\int_{0}^{t} g(\tau) d \tau\right)\|\Delta u\|_{2}^{2}-\frac{1}{p}\|u\|_{p}^{p} \\
& \geq \frac{1}{2}\left\|u_{t}\right\|_{2}^{2}+\frac{1}{2}\left\|\nabla u_{t}\right\|_{2}^{2}+\frac{\eta}{2}\|\Delta u\|_{2}^{2}+\frac{1}{2}(g \circ \Delta u)(t)+\mathcal{Q}\left(\|\nabla u(t)\|_{2}\right) \\
& \geq 0
\end{aligned}
$$


and

$$
\begin{aligned}
E(t) & \leq \frac{1}{2}\left\|u_{t}\right\|_{2}^{2}+\frac{1}{2}\left\|\nabla u_{t}\right\|_{2}^{2}+\frac{1}{2}\|\Delta u\|_{2}^{2}+\frac{1}{2}(g \circ \Delta u)(t)+\frac{1}{2}\|\nabla u\|_{2}^{2} \\
& \leq \frac{1}{2} B^{2}\left\|\nabla u_{t}\right\|_{2}^{2}+\frac{1}{2}\left\|\nabla u_{t}\right\|_{2}^{2}+\frac{1}{2} \lambda_{1}^{-1}\|\Delta u\|_{2}^{2}+\frac{1}{2}\|\Delta u\|_{2}^{2}+\frac{1}{2}(g \circ \Delta u)(t) .
\end{aligned}
$$

Let $C_{1}=\frac{1}{2}\left(1+B^{2}\right)$ and $C_{2}=\frac{1}{2}\left(1+\lambda_{1}^{-1}\right)$, then we have (32).

Lemma 2. The energy $E(t)$ satisfies

$$
\frac{d E(t)}{d t} \leq-\left\|\nabla u_{t}(t)\right\|_{2}^{2}-\frac{1}{2} \xi_{2}(g \circ \Delta u)(t)-\frac{1}{2}\left[g(0)-\xi_{1}\|g\|_{L^{1}(0, \infty)}\right]\|\Delta u(t)\|_{2}^{2} \quad \forall \quad t \geq 0 .
$$

Proof. From (9), we have

$$
\frac{d E(t)}{d t} \leq-\left\|\nabla u_{t}(t)\right\|_{2}^{2}-\frac{\xi_{2}}{2}(g \circ \Delta u)(t)-\frac{1}{2} g(t)\|\Delta u(t)\|_{2}^{2} .
$$

From assumptions (G2) and since $\int_{0}^{t} g^{\prime}(\tau) d \tau=g(t)-g(0)$, we obtain

$$
\begin{aligned}
-\frac{1}{2} g(t)\|\Delta u(t)\|_{2}^{2} & =-\frac{1}{2} g(0)\|\Delta u(t)\|_{2}^{2}-\frac{1}{2}\left(\int_{0}^{t} g^{\prime}(\tau) d \tau\right)\|\Delta u(t)\|_{2}^{2} \\
& \leq-\frac{1}{2} g(0)\|\Delta u(t)\|_{2}^{2}+\frac{\xi_{1}}{2}\|g\|_{L^{1}(0, \infty)}\|\Delta u(t)\|_{2}^{2} \\
& =-\frac{1}{2}\left[g(0)-\xi_{1}\|g\|_{L^{1}(0, \infty)}\right]\|\Delta u(t)\|_{2}^{2} .
\end{aligned}
$$

Then, Combining (34) and (35) our conclusion holds. Multiplying (33) by $e^{\kappa \zeta(t)}(\kappa>0)$ and using (32), we have

$$
\begin{aligned}
\frac{d}{d t}\left(e^{\kappa \zeta(t)} E(t)\right) \leq & -\left\|\nabla u_{t}(t)\right\|_{2}^{2} e^{\kappa \zeta(t)} E(t)-\frac{1}{2} \xi_{2}(g \circ \Delta u)(t) e^{\kappa \zeta(t)} E(t) \\
& -\frac{1}{2}\left[g(0)-\xi_{1}\|g\|_{L^{1}(0, \infty)}\right]\|\Delta u(t)\|_{2}^{2} e^{\kappa \zeta(t)} E(t)+\kappa \zeta_{t}(t) e^{\kappa \zeta(t)} E(t) \\
\leq & -\left[1-\kappa C_{1} \zeta_{t}(t)\right]\left\|\nabla u_{t}(t)\right\|_{2}^{2} e^{\kappa \zeta(t)} E(t)-\frac{1}{2}\left[\xi_{2}-\kappa \zeta_{t}(t)\right](g \circ \Delta u)(t) e^{\kappa \zeta(t)} E(t) \\
& -\frac{1}{2}\left[g(0)-\xi_{1}\|g\|_{L^{1}}-2 C_{2} \kappa \zeta_{t}(t)\right]\|\Delta u(t)\|_{2}^{2} e^{\kappa \zeta(t)} E(t) .
\end{aligned}
$$

Using the fact that $\zeta_{t}$ is decreasing by (8), we conclude that

$$
\begin{aligned}
\frac{d}{d t}\left(e^{\kappa \zeta(t)} E(t)\right) \leq & -\left[1-\kappa C_{1} \zeta_{t}(0)\right]\left\|\nabla u_{t}(t)\right\|_{2}^{2} e^{\kappa \zeta(t)} E(t)-\frac{1}{2}\left[\xi_{2}-\kappa \zeta_{t}(0)\right](g \circ \Delta u)(t) e^{\kappa \zeta(t)} E(t) \\
& -\frac{1}{2}\left[g(0)-\xi_{1}\|g\|_{L^{1}(0, \infty)}-2 C_{2} \kappa \zeta_{t}(0)\right]\|\Delta u(t)\|_{2}^{2} e^{\kappa \zeta(t)} E(t) .
\end{aligned}
$$

Choosing $\|g\|_{L^{1}(0, \infty)}$ sufficiently small so that

$$
g(0)-\xi_{1}\|g\|_{L^{1}(0, \infty)}=K>0
$$

and defining

$$
\kappa_{0}=\min \left\{\frac{1}{C_{1} \zeta_{t}(0)}, \frac{\xi_{2}}{\zeta_{t}(0)}, \frac{K}{2 C_{2} \zeta_{t}(0)}\right\},
$$

we conclude by taking $\kappa \in\left(0, \kappa_{0}\right]$ in (37) that

$$
\frac{d}{d t}\left(e^{\kappa \zeta(t)} E(t)\right) \leq 0, \quad t>0 .
$$


Integrating (38) over $(0, t)$, it follows that

$$
E(t) \leq E(0) e^{-\kappa \zeta(t)}, \quad t>0
$$

Example 1. For $\zeta(t)=t+\frac{t}{t+1}$, we can get the exponential decay rate $E(t) \leq E(0) e^{-\kappa t}, \quad \forall t \geq 0$. For $\zeta(t)=$ $\ln (1+t)$, we can get polynomial decay rate $E(t) \leq E(0)(1+t)^{-\kappa}, \quad \forall t \geq 0$.

Conflicts of Interest: "The author declares no conflict of interest."

\section{References}

[1] Di, H., \& Shang, Y. (2015). Global existence and asymptotic behavior of solutions for the double dispersive-dissipative wave equation with nonlinear damping and source terms. Boundary Value Problems, 2015, Article no. 29.

[2] Shang, Y. D. (2000). Initial boundary value problem of equation $u_{t t}-\Delta u-\Delta u_{t}-\Delta u_{t t}=f(u)$. Acta Mathematicae Applicatae Sinica, 23(3), 385-393.

[3] Zhang, H. W., \& Hu, Q. Y. (2004). Existence of global weak solution and stability of a class nonlinear evolution equation. Acta Mathematicae Applicatae Sinica, 24A(3), 329-336.

[4] Xie, Y., \& Zhong, C. (2007). The existence of global attractors for a class nonlinear evolution equation. Journal of Mathematical Analysis and Applications, 336(1), 54-69.

[5] Xu, R. Z., Zhao, X. R., \& Shen, J. H. (2008). Asymptotic behaviour of solution for fourth order wave equation with dispersive and dissipative terms. Applied Mathematics and Mechanics, 29(2), 259-262.

[6] Mellah, M. (2020). Global solutions and general decay for the dispersive wave equation with memory and source terms. Open Journal of Mathematical Analysis, 4(2), 116-122.

[7] Mellah, M., \& Hakem, A. (2019). Global existence, uniqueness, and asymptotic behavior of solution for the Euler-Bernoulli viscoelastic equation. Open Journal of Mathematical Analysis, 3(1), 42-51.

[8] Park, J. Y., \& Kang, J. R. (2010). Global existence and uniform decay for a nonlinear viscoelastic equation with damping. Acta Applicandae Mathematicae, 110(3), 1393-1406.

[9] Lions, J. L. (1969). Quelques Méthodes de Résolution des Problèmes aux Limites Non Linéaires. Dunod Gauthier-Villars, Paris. 